\title{
RENTABILIDAD DE LOS FONDOS DE PENSIONES 1994-2013
}

\author{
PROFITABILITY OF PENSION FUND 1994-2013 \\ José Salvador Romero Cerna* \\ Docente Auxiliar de la Facultad de Ciencias Contables \\ Universidad Nacional Mayor de San Marcos-UNMSM / Lima-Perú \\ [Recepción: Setiembre de 2014/ Conformidad: Octubre 2014]
}

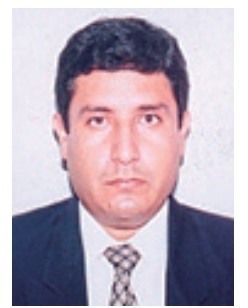

\section{RESUMEN}

Luego de la reforma del Sistema Privado de Pensiones (SPP) y la derogación del aporte obligatorio de los trabajadores independientes al SPP, el debate sobre el sistema previsional se ha centrado en una reforma integral del mismo. Uno de los puntos en discusión es el nivel de rentabilidad que ha presentado los fondos de pensiones administrados por las Administradoras de Fondos de Pensiones (AFP) desde su creación (1993), algunos analistas utilizan las rentabilidades nominales (sin incluir las comisiones que cobran las AFP) publicadas por la Superintendencia de Banca, Seguros y AFP (SBS), y otros utilizan las rentabilidades nominales corregidas (al incluir las comisiones). El artículo estima la rentabilidad nominal promedio anual neta de comisiones durante un período de 20 años (1994-2013), para determinar si esta rentabilidad de los fondos de pensiones ha sido superior a otras alternativas de ahorro en el sistema financiero, y sobre esa base formular políticas públicas.

\section{Palabras clave:}

Reforma del sistema privado de pensiones; rentabilidad nominal neta de comisiones.

\begin{abstract}
After the reform of the Private Pension System (PPS) and the repeal of obligatory contribution by independent workers to SPP, the debate on the pension system has focused on a comprehensive reform of it. One of the points at issue is the level of profitability which has filed the pension funds administered by the Pension Fund Administrators (AFP) since its inception (1993), some analysts use the nominal returns (excluding commissions charged by AFP) published by the Superintendency of Banking and Insurance (SBS), and others use the corrected nominal returns (by including commissions).

The article estimates the average annual net commissions nominal return over a period of 20 years (19942013) to determine whether the return on pension funds has been higher than other savings options in the financial system, and based on that formulate public policies.
\end{abstract}

\section{Keywords:}

Reform of the private pension system; net nominal return from commissions.

\footnotetext{
* Magíster en Economía con mención en Finanzas, UNMSM. E-mail: jromeroc5@unmsm.edu.pe
} 


\section{INTRODUCCIÓN}

Luego de la reciente reforma del Sistema Privado de Pensiones (SPP) y de la eliminación de la obligatoriedad de los aportes de los trabajadores independientes al SPP, uno de los aspectos en que se centró la discusión fue sobre la rentabilidad de los fondos de pensiones administrados por el SPP.

Según el Presidente de la Asociación de Administradoras de Fondos de Pensiones, Luis Miguel Valdivieso Montano, ex-ministro de Economía y Finanzas (07/2008-01/2009), la rentabilidad nominal histórica del fondo 2 de pensiones privado durante los 20 años del SPP es de $12,72 \%$ promedio anual (sin incluir la comisión de administración), y la rentabilidad real ajustada (incluyendo la comisión de administración) es de $7.5 \%$ promedio anual en la modalidad de retiro programado ${ }^{1}$. Por su parte, para el economista Jorge Rojas la rentabilidad real ajustada (incluyendo la comisión de administración) es de 6\% promedio anual ${ }^{2}$.

Lorena Masías, ex-superintendente adjunta de las Administradoras Privadas de Fondos de Pensiones de la SBS (02/1998-09/2011), coincide con Luis Valdivieso en la rentabilidad nominal histórica, al señalar que la rentabilidad promedio de los fondos en los últimos 20 años es de casi $12 \%$ anual publicado en el diario (El Comercio 04/09/2014 p. B3), mientras que el economista Carlos Adrianzén coincide con Jorge Rojas en la rentabilidad real ajustada al señalar que a junio pasado registraba una rentabilidad real por década elevada (6\%) (El Comercio 13/08/2014 p. A21).

\section{RENTABILIDAD NOMINAL NETA DE LOS FONDOS DE PENSIONES 1994-2013}

Para estimar la rentabilidad neta de comisiones durante el período de 20 años de 1994 al 2013, se ha tomado como muestra las rentabilidades nominales acumuladas al 31.12.2013 del fondo 2 publicadas por la $\mathrm{SBS}^{3}$ correspondiente a los valores cuota promedio del mes de diciembre para cada año y se ha calculado, el porcentaje de comisión de administración promedio de las AFP que al 31.12.2013 están operativas (Integra, Profuturo y Prima) 4 .

Para cada aporte efectuado en el mes de diciembre de cada año (desde 1993 hasta el año 2012), se tiene el rendimiento nominal acumulado al 31.12.2013; es decir, para el aporte efectuado en diciembre de 1993 el rendimiento nominal acumulado corresponde a un período de 20 años, para el aporte efectuado en diciembre de 1994 el rendimiento corresponde a 19 años y así; sucesivamente, hasta para el aporte efectuado en diciembre de 2012 el rendimiento corresponde a 1 año.

Como la comisión de administración (comisión por flujo) cobrada por cada aporte implica la prestación del servicio por parte de la Administradora de Fondos de Pensiones (AFP) hasta que los trabajadores se jubilen; esta debe adicionarse al aporte efectuado para calcular la rentabilidad nominal acumulada neta de comisiones.

1 Diario Gestión 17.09.2014.

2 Diario Gestión 15.09.2014. Anteriormente, para el período 1993-1997 Jorge Rojas estimó una rentabilidad nominal neta producida por el SPP de $-0.18 \%$ anual.

3 Asumiendo que las rentabilidades calculadas por la SBS son comparables con otras alternativas de inversión. Habría que evaluar, por ejemplo, el grado en que se reduce la volatilidad de los rendimientos al calcular las rentabilidades en base a valores cuotas promedio, $y$ al valorizar los instrumentos de renta fija (bonos) en base al vector de precios que determina la SBS. En ese sentido, habría que evaluar si es correcta la afirmación del ex-ministro Luis Valdivieso que desde 1993 no ha habido, en el Perú, otro instrumento de inversión más rentable que los fondos de pensiones privados (diario Uno 30/08/2014 p.3).

4 Hasta el 31.12.1993 Integra y Profuturo solo cobraban una comisión fija que se eliminó en enero de 1997. Para determinar la tasa de comisión promedio se ha considerado la comisión sobre flujo (\% sobre remuneración bruta mensual) y se ha omitido la comisión fija cobrada durante el período 1994-1996.

164 / QVIPURAMAYOC|Vol. 22(43) 2014 


\section{Cuadro No 01:}

Rendimiento Nominal Neto de Comisión Promedio Anual Para Aportes por Periodo al 31/12/13

\begin{tabular}{|c|c|c|c|c|c|c|}
\hline \multirow[b]{2}{*}{$\begin{array}{l}\text { Período } \\
\text { Aporte }\end{array}$} & \multirow[b]{2}{*}{$\begin{array}{c}\text { Años al } \\
31 / 12 / 2013\end{array}$} & \multicolumn{2}{|c|}{ Rendimiento Nominal } & \multicolumn{2}{|c|}{ Rend Neto Comisión } & \multirow[b]{2}{*}{$\begin{array}{c}\text { Comisión } \\
\text { Promedio (1) }\end{array}$} \\
\hline & & $\begin{array}{r}\begin{array}{r}\text { Acumulado } \\
31 / 12 / 2013\end{array} \\
\end{array}$ & $\begin{array}{c}\text { Promedio } \\
\text { Anual }\end{array}$ & \begin{tabular}{|r|} 
Acumulado \\
$31 / 12 / 2013$
\end{tabular} & $\begin{array}{c}\text { Promedio } \\
\text { Anual }\end{array}$ & \\
\hline $12 / 93$ & 20 & $949.56 \%$ & $12.47 \%$ & $777.92 \%$ & $11.47 \%$ & $1.96 \%$ \\
\hline $12 / 94$ & 19 & $735.47 \%$ & $11.82 \%$ & $590.47 \%$ & $10.70 \%$ & $2.10 \%$ \\
\hline $12 / 95$ & 18 & $618.18 \%$ & $11.58 \%$ & $497.99 \%$ & $10.45 \%$ & $2.01 \%$ \\
\hline $12 / 96$ & 17 & $501.39 \%$ & $11.13 \%$ & $399.91 \%$ & $9.93 \%$ & $2.03 \%$ \\
\hline $12 / 97$ & 16 & $407.37 \%$ & $10.68 \%$ & $308.35 \%$ & $9.19 \%$ & $2.43 \%$ \\
\hline $12 / 98$ & 15 & $404.37 \%$ & $11.39 \%$ & $287.05 \%$ & $9.44 \%$ & $2.43 \%$ \\
\hline $12 / 99$ & 14 & $311.57 \%$ & $10.63 \%$ & $215.84 \%$ & $8.56 \%$ & $2.43 \%$ \\
\hline $12 / 00$ & 13 & $325.84 \%$ & $11.79 \%$ & $226.78 \%$ & $9.54 \%$ & $2.43 \%$ \\
\hline $12 / 01$ & 12 & $280.73 \%$ & $11.79 \%$ & $192.17 \%$ & $9.35 \%$ & $2.43 \%$ \\
\hline $12 / 02$ & 11 & $237.23 \%$ & $11.68 \%$ & $162.56 \%$ & $9.17 \%$ & $2.28 \%$ \\
\hline $12 / 03$ & 10 & $171.25 \%$ & $10.49 \%$ & $111.19 \%$ & $7.76 \%$ & $2.28 \%$ \\
\hline $12 / 04$ & 9 & $149.33 \%$ & $10.68 \%$ & $94.13 \%$ & $7.65 \%$ & $2.28 \%$ \\
\hline $12 / 05$ & 8 & $107.64 \%$ & $9.56 \%$ & $67.51 \%$ & $6.66 \%$ & $1.92 \%$ \\
\hline $12 / 06$ & 7 & $61.86 \%$ & $7.12 \%$ & $37.63 \%$ & $4.67 \%$ & $1.76 \%$ \\
\hline $12 / 07$ & 6 & $29.43 \%$ & $4.39 \%$ & $10.06 \%$ & $1.61 \%$ & $1.76 \%$ \\
\hline $12 / 08$ & 5 & $64.53 \%$ & $10.47 \%$ & $38.92 \%$ & $6.80 \%$ & $1.84 \%$ \\
\hline $12 / 09$ & 4 & $23.60 \%$ & $5.44 \%$ & $3.43 \%$ & $0.85 \%$ & $1.95 \%$ \\
\hline $12 / 10$ & 3 & $4.12 \%$ & $1.36 \%$ & $-12.87 \%$ & $-4.49 \%$ & $1.95 \%$ \\
\hline $12 / 11$ & 2 & $11.58 \%$ & $5.63 \%$ & $-6.29 \%$ & $-3.19 \%$ & $1.91 \%$ \\
\hline $12 / 12$ & 1 & $-0.04 \%$ & $-0.04 \%$ & $-15.38 \%$ & $-15.38 \%$ & $1.81 \%$ \\
\hline
\end{tabular}

Fuente: SBS. Elaboración: Propia.

(1) Promedio simple de comisión sobre la remuneración (flujo) de Integra, Profuturo y Prima. Prima ingresa en agosto 2005. Para el periodo 12/1993 se ha tomado la comisión de 01/1994.

Para determinar la rentabilidad nominal promedio anual (k) se toma el valor final (VF) calculado sobre el valor (aporte) inicial (VI), y se divide entre el VI más el importe de la comisión (C) durante el período correspondiente, es decir $\mathrm{k}=[\mathrm{VF} /(\mathrm{VI}+\mathrm{C})]$ ^ $(1 / \mathrm{n})-1$. Así, se obtiene un rendimiento nominal promedio anual neto de comisión para períodos de 1 a 20 años, por ejemplo para un período de 20 años (dic 2013 / dic 1993) el rendimiento es de 11.47\%, para 15 años (dic 2013 / dic 1998) es de 9.44\%, para 10 años (dic 2013 / dic 2003) es de $7.76 \%$, y para 5 años (dic 2013 / dic 2008) es de 6.80\%. Esto significa que, por ejemplo para el aporte efectuado en diciembre de $1993^{5}$ el rendimiento nominal promedio anual al 31.12.2013 es de $11.47 \%$, y para el aporte efectuado en diciembre de 2008 es de $6.80 \%$, entonces como cada aporte tendrá un rendimiento acumulado hasta la jubilación. Una forma de estimar el rendimiento promedio nominal anual (neto de comisiones) para el período dic 1993 - dic 2013 es determinando la tasa de rendimiento implícita $(\mathrm{k})$ para una anualidad anticipada o prepagable ${ }^{6}$ (aporte al fondo por cada período) durante 20 años y un valor final al 31.12.2013 determinado en base a los rendimientos obtenidos por cada anualidad (aporte).

$$
\mathrm{VF}_{\mathrm{n}}=\mathrm{Qx} \frac{(1+\mathrm{k})^{\mathrm{n}}-1}{\mathrm{k}} \mathrm{x}(1+\mathrm{k})
$$

Así, para una cuota (aporte) de 1 (Q) durante 20 años (n) y un valor final (VF) al 31.12.2013 de 59.87 (obtenido en base a los rendimientos neto de comisiones estimados) se tiene una tasa implícita promedio anual de rendimiento (k) de 9.58\% (1994-2013).

\footnotetext{
5 Para el determinar la rentabilidad acumulada (incluyendo comisiones) al 31.12.2013 del aporte efectuado en diciembre de 1993 se considera la tasa de comisión correspondiente a enero 1994 ya que durante el año 1993 las comisiones consistían en una comisión fija. 6 Ver El valor temporal del dinero, monografía de Juan Mascareñas, mayo 2013.

http://papers.ssrn.com/sol3/papers.cfm?abstract_id=2312111
} 


\section{RENTABILIDAD NOMINAL NETA PROMEDIO ANUAL DEL FONDO TIPO 2 ADMINISTRADO POR INTEGRA Y PROFUTURO (ENERO 1994 / ENERO 2014)}

El mismo ejercicio se efectuó tomando los valores cuota promedio históricos mensuales del fondo tipo 2 de Integra y Profuturo para el período enero 1994 a enero 2014, publicados por la SBS.

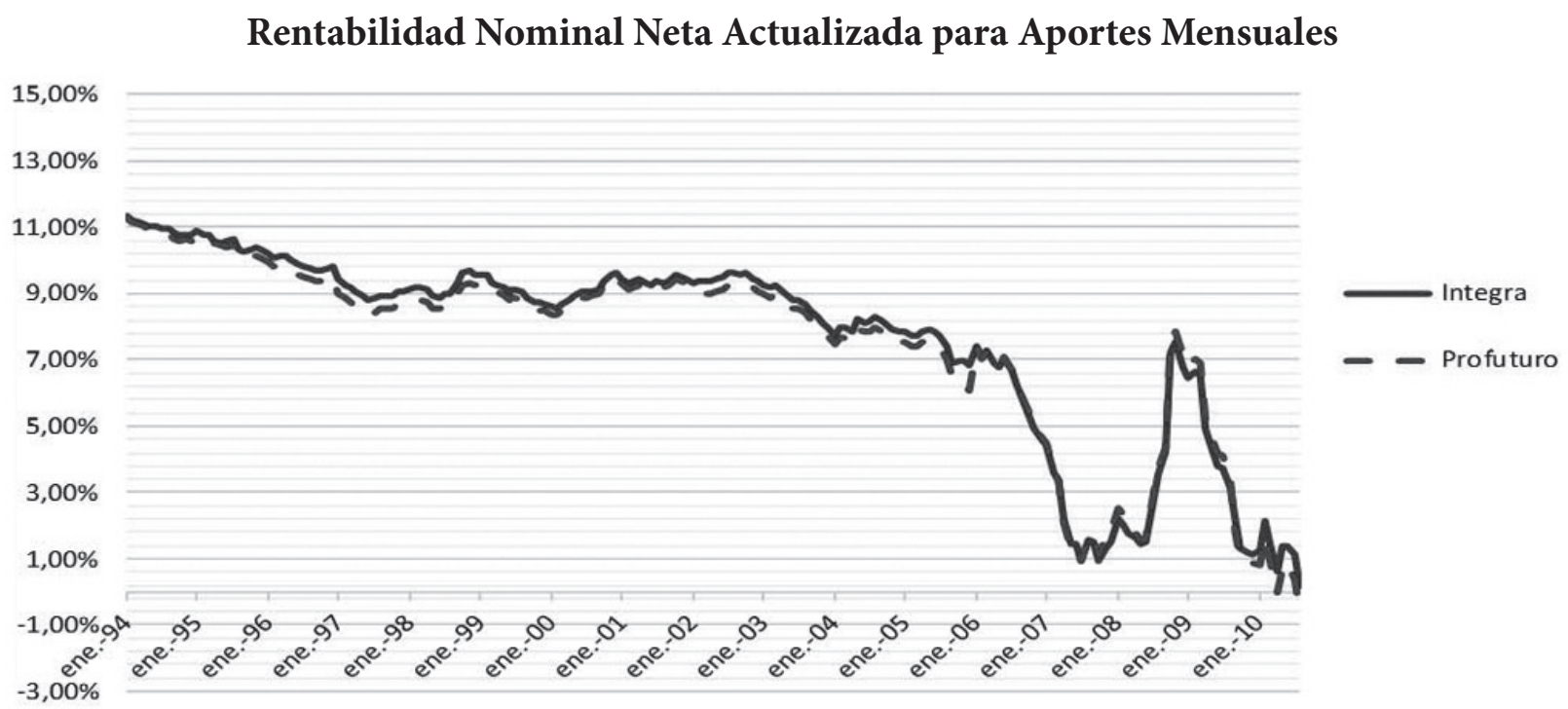

Fuente: S.B.S. Elaboración: Propia.

Así, para 240 cuotas (aportes mensuales) se tiene una tasa implícita promedio anual de rendimiento $(\mathrm{k})$ de $9.29 \%$ y $9.04 \%$ para Integra y Profuturo, respectivamente. Es decir, tomando los valores cuotas mensuales la rentabilidad promedio anual es de $9.16 \%$ para el período enero 1994 - enero 2014.

Las rentabilidades nominales netas de comisiones anualizadas para los períodos mensuales mostradas en el gráfico presentan la misma tendencia decreciente que para la muestra de aportes anuales, rentabilidades que se tornan negativos a partir del período $09 / 2010$.

\section{CONCLUSIONES}

1. Se ha estimado la rentabilidad nominal promedio anual neta de comisiones durante el período de 20 años (enero 1994 - enero 2014), tomando los valores cuota promedio del fondo tipo 2 (de Integra y Profuturo) publicados por la SBS, determinando la tasa de rendimiento implícita $(\mathrm{k})$ para una anualidad anticipada o prepagable, en aproximadamente $9.16 \%$. Asumiendo una tasa de inflación de $3 \%$ anual se tiene una rentabilidad real neta de comisiones del orden de $6 \%$, una tasa similar a la señalada por el economista Jorge Rojas.

2. La rentabilidad nominal promedio anual neta de comisiones de aproximadamente $9.16 \%$ no es la alternativa de ahorro que genera mayores ganancias (rendimientos). Según la Asociación de AFP los fondos de pensiones administrados por las AFP han dado mayores ganancias que los fondos mutuos, depósitos de CTS en cajas municipales, rurales o bancos?.

3. La propuesta sobre la Reforma del SPP y del Sistema Previsional debe ser un tema central en las próximas elecciones presidenciales, donde se determinen la tasa de aporte considerando el ciclo de ingresos, la tasa de reemplazo, el establecimiento de una pensión universal no contributiva a cargo del presupuesto público, el ingreso de otras instituciones financieras (bancos, cajas, etc.) al sistema previsional, etc.

7 Según la Asociación de AFP la rentabilidad nominal del fondo tipo 2 es de 12.7\% por año para un período de 21 años (agosto 1993 julio 2014).

166/ QVIPURAMAYOC|Vol. 22(43) 2014 


\section{REFERENCIAS BIBLIOGRÁFICAS}

1. ASOCIACIÓN DE AFP (2013) Portal electrónico http://www.asociacionafp.com.pe

2. MASCAREÑAS, Juan (2013) El valor temporal del dinero. http://papers.ssrn.com/sol3/papers. cfm?abstract_id $=2312111$
3. ROJAS, Jorge (1998) La Rentabilidad del Sistema Privado de Pensiones en el Perú: 1993-1997. PUCP.

http://departamento.pucp.edu.pe/economia/ images/documentos/DDD160.pdf

4. SBS. http://www.sbs.gob.pe 